MATHEMATICS OF COMPUTATION

Volume 73, Number 245, Pages 1-18

S $0025-5718(03) 01520-5$

Article electronically published on March 27, 2003

\title{
NONCONFORMING ELEMENTS IN LEAST-SQUARES MIXED FINITE ELEMENT METHODS
}

\author{
HUO-YUAN DUAN AND GUO-PING LIANG
}

\begin{abstract}
In this paper we analyze the finite element discretization for the first-order system least squares mixed model for the second-order elliptic problem by means of using nonconforming and conforming elements to approximate displacement and stress, respectively. Moreover, on arbitrary regular quadrilaterals, we propose new variants of both the rotated $\mathcal{Q}_{1}$ nonconforming element and the lowest-order Raviart-Thomas element.
\end{abstract}

\section{INTRODUCTION}

As is well-known, nonconforming elements (e.g., Crouzeix-Raviart (CR) linear elements [11] and the rotated $\mathcal{Q}_{1}$-element [12], [18, [10]) are very useful to seek numerical solutions of many physical problems (see [11], 12], 113, [15], [16, [17, [18], [27, [10]). A quadrilateral version of the rotated $\mathcal{Q}_{1}$-element was studied in [18], but it is only suitable for uniform asymptotic rectangles. This is a restrictive condition. In this paper, we propose a new variant which admits arbitrary regular quadrilaterals and allows the finite element equation to be efficiently obtained on the reference element.

In the classical mixed finite element analysis, both triangular and rectangular normal continuous elements 19 are proposed, which are known as Raviart-ThomasNédélec (RTN) elements [5, 8], 9] and Brezzi-Douglas-Marini (BDM) elements [7] and Brezzi-Douglas-Fortin-Marini (BDFM) elements [6], and so on. On arbitrary quadrilaterals, Wang and Mathew [25] analyzed variants of these elements, but the very important commuting diagram property does not hold (cf. [35], [19], [37]). In this paper, we propose a new variant of the lowest-order RTN rectangular element. Our variant is the first one which not only admits arbitrary regular quadrilaterals, but also satisfies the commuting diagram property.

These above two new elements will be used for the finite element discretization of the first-order system least-squares mixed model for a second-order elliptic problem with various boundary conditions.

It is well known that one advantage of the least squares mixed method 4 is that coerciveness holds, while the classical mixed method [19], 28 is subject to the Babuška-Brezzi condition. However, it seems that the coerciveness strongly depends on the conformity of the finite dimensional spaces (see 1], 2], [3], 23], [24],

Received by the editor May 29, 2001 and, in revised form, May 7, 2002.

2000 Mathematics Subject Classification. Primary 65N30.

Key words and phrases. Second-order elliptic problem, least-squares mixed finite element method, nonconforming element, normal continuous element. 
[26]). Up to now, it is not clear whether the coerciveness still holds if the displacement is approximated by nonconforming elements and the stress by conforming elements. In this paper, on triangular, rectangular and quadrilateral meshes, nonconforming finite element methods are analyzed in a unified way. It is shown that our nonconforming methods are still coercive, and optimal error bounds are derived.

As is known, the so-called inconsistent error is an essential feature of the nonconforming displacement-based finite element method [20, 29], 22]. In this paper, we find that this error does not exist in the first-order system least-squares mixed methods in the case of nonconforming elements. It seems that the theory of the patch test [20], 29], 30] would be lost. Nonetheless, it turns out that the patch test is necessary to obtain coerciveness.

The rest of the paper is arranged as follows. In section 2, the first-order system least-squares mixed model is recalled for the second order elliptic problem. In section 3, nonconforming finite element methods are analyzed for the least-squares mixed model. In section 4 , two quadrilateral elements are proposed. In section 5 , some comments are made.

\section{The LEAST-SQUARES MIXED MODEL}

Let $\Omega \subset \Re^{d}(d=2,3)$ be a bounded domain with Lipschitz boundary $\Gamma=\partial \Omega$. Given a subdomain $O \subseteq \Omega$ with Lipschitz boundary $\partial O$, we introduce $L^{2}(O)$, and $\left(L^{2}(O)\right)^{d}$, with inner product $(\cdot, \cdot)_{0, O}$ and norm $\|\cdot\|_{0, O}$, and introduce $L^{2}$-based Sobolev spaces $H^{m}(O)$ and $\left(H^{m}(O)\right)^{d}$, with norm $\|\cdot\|_{m, O}$ and semi-norm $|\cdot|_{m, O}$ $\left(m \geq 1\right.$ is an integer). In addition, we introduce $H_{0}^{1}(O)=\left\{v \in H^{1}(O) ; v_{\left.\right|_{\text {əo }}}=0\right\}$ with norm $|\cdot|_{1, O}$, and $H(\operatorname{div} ; O)=\left\{\boldsymbol{q} \in\left(L^{2}(O)\right)^{d} ; \operatorname{div} \boldsymbol{q} \in L^{2}(O)\right\}$ with norm $\|\cdot\|_{H(\operatorname{div} ; O)}($ cf. [34]).

In the case $O=\Omega$, we simplify the notation as follows: $|\cdot|_{m, O} \equiv|\cdot|_{m},\|\cdot\|_{m, O} \equiv$ $\|\cdot\|_{m}(m \geq 1),(\cdot, \cdot)_{0, O} \equiv(\cdot, \cdot),\|\cdot\|_{0, O} \equiv\|\cdot\|$.

Let $\Gamma=\Gamma_{D} \cup \Gamma_{N}$ with $\Gamma_{D} \cap \Gamma_{N}=\emptyset$, and let $\boldsymbol{n}$ be the unit outward normal vector to $\Gamma$. We additionally introduce

$$
\begin{gathered}
H_{0, D}^{1}(\Omega)=\left\{v \in H^{1}(\Omega) ; v=0 \quad \text { on } \Gamma_{D}\right\}, \\
H_{0, N}(\operatorname{div} ; \Omega)=\left\{\boldsymbol{q} \in H(\operatorname{div} ; \Omega) ; \boldsymbol{q} \cdot \boldsymbol{n}=0 \text { on } \Gamma_{N}\right\}, \\
H^{1}(\operatorname{div} ; \Omega)=\left\{\boldsymbol{q} \in\left(H^{1}(\Omega)\right)^{d} ; \operatorname{div} \boldsymbol{q} \in H^{1}(\Omega)\right\} .
\end{gathered}
$$

Considering the following second-order elliptic problem:

$$
\begin{gathered}
-\sum_{i, j=1}^{d} \frac{\partial}{\partial x_{i}}\left(a_{i j} \frac{\partial u}{\partial x_{j}}\right)=f \quad \text { in } \Omega, \\
u=0 \quad \text { on } \Gamma_{D}, \\
-\sum_{i, j=1}^{d} n_{i} a_{i j} \frac{\partial u}{\partial x_{j}}=0 \quad \text { on } \Gamma_{N},
\end{gathered}
$$

where $u$ is the displacement and $A=\left(a_{i j}(x)\right) \in \Re^{d \times d}$ is a sufficiently smooth, symmetric matrix of coefficients, satisfying

$$
C \sum_{i=1}^{d} \xi_{i}^{2} \leq \sum_{i, j=1}^{d} a_{i j}(x) \xi_{i} \xi_{j} \leq C^{-1} \sum_{i=1}^{d} \xi_{i}^{2} \quad \forall\left(\xi_{i}\right) \in \Re^{d}, \forall x \in \bar{\Omega} .
$$


Here and below, the letter $C$ (with or without subscripts) is a generic constant which may take different values at different occurrences.

Introducing the stress

$$
\boldsymbol{p}=-A \nabla u
$$

as an independent variable, we can rewrite (2.1) in the first order system

$$
\begin{gathered}
\operatorname{div} \boldsymbol{p}=f, \quad \boldsymbol{p}=-A \nabla u \quad \text { in } \Omega \\
u=0 \quad \text { on } \Gamma_{D}, \\
\boldsymbol{p} \cdot \boldsymbol{n}=0 \quad \text { on } \Gamma_{N} .
\end{gathered}
$$

A least-squares variational problem for (2.4) is to find $u \in U=H_{0, D}^{1}(\Omega)$ and $\boldsymbol{p} \in X=H_{0, N}(\operatorname{div} ; \Omega)$ such that

$$
L(u, \boldsymbol{p} ; v, \boldsymbol{q}):=(\boldsymbol{p}+A \nabla u, \boldsymbol{q}+A \nabla v)+(\operatorname{div} \boldsymbol{p}, \operatorname{div} \boldsymbol{q})=(f, \operatorname{div} \boldsymbol{q})
$$

for all $(v, \boldsymbol{q}) \in U \times X$.

\section{The NONCONFORMing Finite ELEMENT METHod}

3.1. Method (I). Let $\mathcal{T}_{h}$ be the regular triangulation [20, 22] of $\Omega$ into triangles or rectangles in $\Re^{2}$, or tetrahedra or rectangular solids in $\Re^{3}$. We define

$$
U_{h} \not \subset U, \quad X_{h} \subset X .
$$

A finite element method for problem $(2.5)$ is to find $\left(u_{h}, \boldsymbol{p}_{h}\right) \in U_{h} \times X_{h}$ such that

$$
\left\{\begin{array}{l}
L_{h}\left(u_{h}, \boldsymbol{p}_{h} ; v_{h}, \boldsymbol{q}_{h}\right) \\
:=\left(\boldsymbol{p}_{h}+A \nabla_{h} u_{h}, \boldsymbol{q}_{h}+A \nabla_{h} v_{h}\right)+\left(\operatorname{div} \boldsymbol{p}_{h}, \operatorname{div} \boldsymbol{q}_{h}\right)=\left(f, \operatorname{div} \boldsymbol{q}_{h}\right) \\
\forall\left(v_{h}, \boldsymbol{q}_{h}\right) \in U_{h} \times X_{h},
\end{array}\right.
$$

where

$$
\nabla_{h} \text { is the gradient operator element-by-element. }
$$

To investigate both coerciveness and convergence, we define

$$
|\cdot|_{1, h}=\sqrt{\sum_{K \in \mathcal{T}_{h}}|\cdot|_{1, K}^{2}}, \quad\|\cdot\|_{1, h}=\sqrt{\|\cdot\|^{2}+|\cdot|_{1, h}^{2}} .
$$

Hypothesis (H1). The equality

$$
\sum_{K \in \mathcal{T}_{h}} \int_{\partial K} \boldsymbol{q}_{h} \cdot \boldsymbol{n}_{K} v_{h}=0 \quad \forall\left(v_{h}, \boldsymbol{q}_{h}\right) \in U_{h} \times X_{h}
$$

holds, where $\boldsymbol{n}_{K}$ is the unit outward normal vector to $K$ with boundary $\partial K$.

Hypothesis (H2). For $u \in U \cap H^{2}(\Omega)$ and $\boldsymbol{p} \in X \cap H^{1}$ (div; $\Omega$ ), there exist two interpolants $I_{h} u \in U_{h}$ and $\Pi_{h} \boldsymbol{p} \in X_{h}$ such that

$$
\begin{gathered}
\left\|u-I_{h} u\right\|+h\left|u-I_{h} u\right|_{1, h} \leq C h^{2}\|u\|_{2}, \\
\left\|\boldsymbol{p}-\Pi_{h} \boldsymbol{p}\right\| \leq C h|\boldsymbol{p}|_{1}, \quad\left\|\operatorname{div}\left(\boldsymbol{p}-\Pi_{h} \boldsymbol{p}\right)\right\| \leq C h|\operatorname{div} \boldsymbol{p}|_{1} .
\end{gathered}
$$

Hypothesis (H3). There exists a constant $C_{1}>0$, independent of $h$, such that

$$
\left\|v_{h}\right\| \leq C_{1}\left|v_{h}\right|_{1, h}, \quad \forall v_{h} \in U_{h} .
$$


Theorem 3.1. Under Hypotheses (H1) and (H3), there exists a constant $C>0$, independent of $h$, such that

$$
L_{h}\left(v_{h}, \boldsymbol{q}_{h} ; v_{h}, \boldsymbol{q}_{h}\right) \geq C\left\{\left\|v_{h}\right\|_{1, h}^{2}+\left\|\boldsymbol{q}_{h}\right\|_{H(\operatorname{div} ; \Omega)}^{2}\right\} \quad \forall\left(v_{h}, \boldsymbol{q}_{h}\right) \in U_{h} \times X_{h} .
$$

Proof. Given a constant $\alpha>0$, we have

$$
\left\|\operatorname{div} \boldsymbol{q}_{h}\right\|^{2}=\left\|\operatorname{div} \boldsymbol{q}_{h}-\alpha v_{h}\right\|^{2}+2 \alpha\left(\operatorname{div} \boldsymbol{q}_{h}, v_{h}\right)-\alpha^{2}\left\|v_{h}\right\|^{2},
$$

where, in the light of Hypothesis (H1), we have

$$
\begin{aligned}
\left(\operatorname{div} \boldsymbol{q}_{h}, v_{h}\right) & =-\sum_{K \in \mathcal{T}_{h}}\left(\boldsymbol{q}, \nabla v_{h}\right)_{0, K}+\sum_{K \in \mathcal{T}_{h}} \int_{\partial K} \boldsymbol{q}_{h} \cdot \boldsymbol{n}_{K} v_{h} \\
& =-\sum_{K \in \mathcal{T}_{h}}\left(\boldsymbol{q}, \nabla v_{h}\right)_{0, K} .
\end{aligned}
$$

Since

$$
\begin{aligned}
& -2 \alpha \sum_{K \in \mathcal{T}_{h}}\left(\boldsymbol{q}, \nabla v_{h}\right)_{0, K}+\left\|\boldsymbol{q}_{h}+A \nabla_{h} v_{h}\right\|^{2} \\
& \quad=\left\|\boldsymbol{q}_{h}+(A-\alpha E) \nabla_{h} v_{h}\right\|^{2}+2 \alpha\left(A \nabla_{h} v_{h}, \nabla_{h} v_{h}\right)-\alpha^{2}\left|v_{h}\right|_{1, h}^{2},
\end{aligned}
$$

where $E$ is the identity matrix, by $(2.2)$ we have

$$
\left(A \nabla_{h} v_{h}, \nabla_{h} v_{h}\right) \geq C_{2}\left|v_{h}\right|_{1, h}^{2} .
$$

Therefore, from (3.10)-(3.13) and Hypothesis (H3) we get

$$
\begin{aligned}
L_{h}\left(v_{h}, \boldsymbol{q}_{h} ; v_{h}, \boldsymbol{q}_{h}\right)= & \left\|\boldsymbol{q}_{h}+A \nabla_{h} v_{h}\right\|^{2}+\left\|\operatorname{div} \boldsymbol{q}_{h}\right\|^{2} \\
= & \left\|\operatorname{div} \boldsymbol{q}_{h}-\alpha v_{h}\right\|^{2}+\left\|\boldsymbol{q}_{h}+(A-\alpha E) \nabla_{h} v_{h}\right\|^{2} \\
& \quad-\alpha^{2}\left\|v_{h}\right\|^{2}+2 \alpha\left(A \nabla_{h} v_{h}, \nabla_{h} v_{h}\right)-\alpha^{2}\left|v_{h}\right|_{1, h}^{2} \\
\geq & \alpha\left(2 C_{2}-\alpha\left(1+C_{1}^{2}\right)\right)\left|v_{h}\right|_{1, h}^{2} .
\end{aligned}
$$

Putting $\alpha=\frac{C_{2}}{1+C_{1}^{2}}$, we get

$$
\begin{gathered}
\alpha\left(2 C_{2}-\alpha\left(1+C_{1}^{2}\right)\right)=\frac{C_{2}^{2}}{1+C_{1}^{2}}, \\
L_{h}\left(v_{h}, \boldsymbol{q}_{h} ; v_{h}, \boldsymbol{q}_{h}\right) \geq C\left|v_{h}\right|_{1, h}^{2},
\end{gathered}
$$

from which we can obtain (3.9), using (3.8) and the triangle inequality.

Theorem 3.2. Let $(u, \boldsymbol{p}=-A \nabla u) \in\left(U \cap H^{2}(\Omega)\right) \times\left(X \cap H^{1}(\operatorname{div} ; \Omega)\right)$ be the exact solutions, and let $\left(u_{h}, \boldsymbol{p}_{h}\right) \in U_{h} \times X_{h}$ be the finite element solution of (3.2). Under Hypotheses (H1)-(H3), we have

$$
\left\|u-u_{h}\right\|_{1, h}+\left\|\boldsymbol{p}-\boldsymbol{p}_{h}\right\|_{H(\operatorname{div} ; \Omega)} \leq C h\left\{\|u\|_{2}+|\operatorname{div} \boldsymbol{p}|_{1}\right\} .
$$


Proof. For any $v_{h} \in U_{h}, \boldsymbol{q}_{h} \in X_{h}$, we have

$$
\begin{aligned}
\| u- & u_{h}\left\|_{1, h}+\right\| \boldsymbol{p}-\boldsymbol{p}_{h} \|_{H(\operatorname{div} ; \Omega)} \\
\leq & \left\|u-v_{h}\right\|_{1, h}+\left\|\boldsymbol{p}-\boldsymbol{q}_{h}\right\|_{H(\operatorname{div} ; \Omega)} \\
& +\left\|v_{h}-u_{h}\right\|_{1, h}+\left\|\boldsymbol{q}_{h}-\boldsymbol{p}_{h}\right\|_{H(\operatorname{div} ; \Omega)},
\end{aligned}
$$

where

$$
\begin{aligned}
\| v_{h}- & u_{h}\left\|_{1, h}^{2}+\right\| \boldsymbol{q}_{h}-\boldsymbol{p}_{h} \|_{H(\mathrm{div} ; \Omega)}^{2} \\
\leq & L_{h}\left(v_{h}-u_{h}, \boldsymbol{q}_{h}-\boldsymbol{p}_{h} ; v_{h}-u_{h}, \boldsymbol{q}_{h}-\boldsymbol{p}_{h}\right) \\
= & L_{h}\left(v_{h}-u, \boldsymbol{q}_{h}-\boldsymbol{p} ; v_{h}-u_{h}, \boldsymbol{q}_{h}-\boldsymbol{p}_{h}\right) \\
& \quad+L_{h}\left(u-u_{h}, \boldsymbol{p}-\boldsymbol{p}_{h} ; v_{h}-u_{h}, \boldsymbol{q}_{h}-\boldsymbol{p}_{h}\right)
\end{aligned}
$$

and

$$
\begin{aligned}
L_{h}\left(u, \boldsymbol{p} ; v_{h}-u_{h}, \boldsymbol{q}_{h}-\boldsymbol{p}_{h}\right) & \\
= & \left(\boldsymbol{p}+A \nabla u, \boldsymbol{q}_{h}-\boldsymbol{p}_{h}+A \nabla_{h}\left(v_{h}-u_{h}\right)\right) \\
& +\left(\operatorname{div} \boldsymbol{p}, \operatorname{div}\left(\boldsymbol{q}_{h}-\boldsymbol{p}_{h}\right)\right) \\
= & \left(f, \operatorname{div}\left(\boldsymbol{q}_{h}-\boldsymbol{p}_{h}\right)\right) \\
= & L_{h}\left(u_{h}, \boldsymbol{p}_{h} ; v_{h}-u_{h}, \boldsymbol{q}_{h}-\boldsymbol{p}_{h}\right) .
\end{aligned}
$$

Hence, from (3.19) and (3.20) we get

$$
\left\|v_{h}-u_{h}\right\|_{1, h}+\left\|\boldsymbol{q}_{h}-\boldsymbol{p}_{h}\right\|_{H(\operatorname{div} ; \Omega)} \leq C\left\{\left\|v_{h}-u\right\|_{1, h}+\left\|\boldsymbol{q}_{h}-\boldsymbol{p}\right\|_{H(\operatorname{div} ; \Omega)}\right\} .
$$

Combining (3.18) and (3.21), from (3.6) and (3.7) we have

$$
\begin{aligned}
& \left\|u-u_{h}\right\|_{1, h}+\left\|\boldsymbol{p}-\boldsymbol{p}_{h}\right\|_{H(\operatorname{div} ; \Omega)} \\
& \quad \leq C \inf _{\left(v_{h}, \boldsymbol{q}_{h}\right) \in U_{h} \times X_{h}}\left\{\left\|u-v_{h}\right\|_{1, h}+\left\|\boldsymbol{p}-\boldsymbol{q}_{h}\right\|_{H(\mathrm{div} ; \Omega)}\right\} \\
& \quad \leq C h\left\{\|u\|_{2}+|\operatorname{div} \boldsymbol{p}|_{1}\right\} .
\end{aligned}
$$

Remark 3.1. Clearly, the standard argument of analyzing nonconforming error is lost in proving Theorem 3.2, since the term of inconsistent error does not exist. Nevertheless, the patch test (i.e., Hypothesis (H1)) is still indispensable to obtain the coerciveness.

Remark 3.2. Let $\Omega$ be a polygonal (or polyhedral) bounded domain, with $\Omega=$ $\bigcup_{K \in \mathcal{T}_{h}} K$. Let $S$ be the set of all element-edges (or faces) in the triangulation $\mathcal{T}_{h}$ and let $S^{0}$ be the set of all internal element-edges (or faces). Let $S^{\partial}=S-S^{0}$ and $S_{D}^{\partial}$ be the set of element-edges (or faces) on $\Gamma_{D}$, and let $S_{N}^{\partial}=S^{\partial}-S_{D}^{\partial}$. Denote by $[v]=v_{\left.\right|_{1}}-v_{\left.\right|_{K_{2}}}$ the jump of $v$ across the interelement boundaries.

Moreover, let $U(K)$ be the nonconforming element of the CR linear element or the rotated $\mathcal{Q}_{1}$ element, and let $X(K)$ be the normal continuous element of the $R T N_{0}(K)$ triangular element or $R T N_{[0]}(K)$ rectangular element of lowest order. 
Define

$$
\begin{gathered}
U_{h}=\left\{v \in L^{2}(\Omega) ; v_{\left.\right|_{K}} \in U(K), \forall K \in \mathcal{T}_{h},\right. \\
\left.\int_{e}[v]=0, \forall e \in S^{0}, \int_{e} v=0, \forall e \in S_{D}^{\partial}\right\}, \\
X_{h}=\left\{\boldsymbol{q} \in X ; \boldsymbol{q}_{\left.\right|_{K}} \in X(K), \forall K \in \mathcal{T}_{h}\right\} .
\end{gathered}
$$

Then Hypotheses (H1) and (H2) hold, while (H3) can be derived from (H1) (see [27]).

3.2. Method (II). If $X_{h}$ is replaced by

$$
X_{h}^{c} \subset X \cap\left(H^{1}(\Omega)\right)^{d},
$$

which is a continuous subspace in the usual sense [20], 22], then it is not obvious whether Theorem 3.1 holds or not, since Hypothesis (H1) is not necessarily valid.

In order to show that Theorem 3.1 is still true with $X_{h}$ and $U_{h}$ given by (3.25) and (3.1), respectively, we replace Hypothesis (H1) by Hypothesis ( $\left.\mathrm{H}^{\prime}\right)$ and give an additional Hypothesis (H4) as follows:

Hypothesis $\left(H 1^{\prime}\right)$. There exists a constant $C>0$, independent of $h$, such that

$$
\left|\sum_{K \in \mathcal{T}_{h}} \int_{\partial K} v_{h} \boldsymbol{\chi} \cdot \boldsymbol{n}_{K}\right| \leq C h\|\boldsymbol{\chi}\|_{1}\left|v_{h}\right|_{1, h} \quad \forall\left(v_{h}, \boldsymbol{\chi}\right) \in U_{h} \times\left(X \cap\left(H^{1}(\Omega)\right)^{d}\right) .
$$

Hypothesis (H4). There exists an interpolation operator $I_{h}^{c}:\left(H^{1}(\Omega)\right)^{d} \rightarrow X_{h}^{c}$ such that

$$
\left\|\left.\left|I_{h}^{c} \chi-\chi \| \leq C h\right| \chi\right|_{1}, \quad\left|I_{h}^{c} \chi\right|_{1} \leq C|\chi|_{1} .\right.
$$

If additionally $\chi \in\left(H^{2}(\Omega)\right)^{d}$, then

$$
\left\|I_{h}^{c} \chi-\chi\right\|+h\left|I_{h}^{c} \chi-\chi\right|_{1} \leq C h^{2}\|\chi\|_{2} .
$$

Theorem 3.3. Under Hypotheses (H1') and (H4), we have

$$
\left\|\boldsymbol{q}_{h}+A \nabla_{h} v_{h}\right\|^{2} \geq C\left\{\left\|\boldsymbol{q}_{h}\right\|^{2}+\left\|v_{h}\right\|_{1, h}^{2}\right\} \quad \forall\left(\boldsymbol{q}_{h}, v_{h}\right) \in X_{h}^{c} \times U_{h},
$$

so long as $h$ is sufficiently small.

Proof. For any $s_{h} \in X_{h}^{c}$, we have

$$
\begin{aligned}
\left\|\boldsymbol{s}_{h}+A \nabla_{h} v_{h}\right\|^{2}=\| & \boldsymbol{s}_{h}+(A-\alpha E) \nabla_{h} v_{h} \|^{2}+2 \alpha\left(\boldsymbol{s}_{h}, \nabla_{h} v_{h}\right) \\
& +2 \alpha\left(A \nabla_{h} v_{h}, \nabla_{h} v_{h}\right)-\alpha^{2}\left|v_{h}\right|_{1, h}^{2},
\end{aligned}
$$

where $\alpha>0$ is a constant to be specified. Choosing $\boldsymbol{q}^{*} \in\left(X \cap\left(H^{1}(\Omega)\right)^{d}\right)$ such that

$$
\operatorname{div} \boldsymbol{q}^{*}=-v_{h}, \quad\left\|\boldsymbol{q}^{*}\right\|_{1} \leq C\left\|v_{h}\right\|,
$$

we have

$$
2 \alpha\left(\boldsymbol{s}_{h}, \nabla_{h} v_{h}\right)=2 \alpha\left(\boldsymbol{s}_{h}-\boldsymbol{q}^{*}, \nabla_{h} v_{h}\right)+2 \alpha\left(\boldsymbol{q}^{*}, \nabla_{h} v_{h}\right),
$$


where, from (3.31) and Hypothesis (H1')

$$
\begin{aligned}
2 \alpha\left(\boldsymbol{q}^{*}, \nabla_{h} v_{h}\right) & =-2 \alpha\left(\operatorname{div} \boldsymbol{q}^{*}, v_{h}\right)+2 \alpha \sum_{K \in \mathcal{C}_{h}} \int_{\partial K} \boldsymbol{q}^{*} \cdot \boldsymbol{n}_{K} v_{h} \\
& \geq 2 \alpha\left\|v_{h}\right\|^{2}-2 \alpha C h\left\|\boldsymbol{q}^{*}\right\|_{1}\left|v_{h}\right|_{1, h} \\
& \geq 2 \alpha\left\{C_{3}\left\|v_{h}\right\|^{2}-C_{4} h^{2}\left|v_{h}\right|_{1, h}^{2}\right\} .
\end{aligned}
$$

Since

$$
2 \alpha\left(A \nabla_{h} v_{h}, \nabla_{h} v_{h}\right)-\alpha^{2}\left|v_{h}\right|_{1, h}^{2} \geq \alpha\left(2 C_{2}-\alpha\right)\left|v_{h}\right|_{1, h}^{2},
$$

then, if we put $0<\alpha<2 C_{2}$, we have

$$
\begin{aligned}
\left\|\boldsymbol{s}_{h}+A \nabla_{h} v_{h}\right\|^{2} \geq & \left\|\boldsymbol{s}_{h}+(A-\alpha E) \nabla_{h} v_{h}\right\|^{2}+\alpha\left(2 C_{2}-\alpha\right)\left|v_{h}\right|_{1, h}^{2} \\
& +2 \alpha C_{3}\left\|v_{h}\right\|^{2}-2 \alpha C_{4} h^{2}\left|v_{h}\right|_{1, h}^{2}-2 \alpha \| \boldsymbol{s}_{h}-\boldsymbol{q}^{*}||\left|v_{h}\right|_{1, h} .
\end{aligned}
$$

Then, taking the infimum in (3.35) with respect to $s_{h}$, we have

$$
\begin{gathered}
\inf _{\boldsymbol{s}_{h} \in X_{h}^{c}}\left\|\boldsymbol{s}_{h}+A \nabla_{h} v_{h}\right\|^{2}+2 \alpha\left|v_{h}\right|_{1, h} \inf _{\boldsymbol{s}_{h} \in X_{h}^{c}}\left\|\boldsymbol{s}_{h}-\boldsymbol{q}^{*}\right\| \\
\geq 2 \alpha C_{3}\left\|v_{h}\right\|^{2}+\alpha\left(2 C_{2}-\alpha-2 C_{4} h^{2}\right)\left|v_{h}\right|_{1, h}^{2} .
\end{gathered}
$$

It follows that

$$
\begin{aligned}
\operatorname{sinf}_{h \in X_{h}^{c}} & \left\|\boldsymbol{s}_{h}+A \nabla_{h} v_{h}\right\|^{2} \\
\geq & -2 \alpha\left|v_{h}\right|_{1, h} \inf _{\boldsymbol{s}_{h} \in X_{h}^{c}}\left\|\boldsymbol{s}_{h}-\boldsymbol{q}^{*}\right\| \\
& +2 \alpha C_{3}\left\|v_{h}\right\|^{2}+\alpha\left(2 C_{2}-\alpha-2 C_{4} h^{2}\right)\left|v_{h}\right|_{1, h}^{2} \\
\geq & -2 \alpha\left|v_{h}\right|_{1, h} \| I_{h}^{c} \boldsymbol{q}^{*}-\boldsymbol{q}^{*}|| \\
& +2 \alpha C_{3}\left\|v_{h}\right\|^{2}+\alpha\left(2 C_{2}-\alpha-2 C_{4} h^{2}\right)\left|v_{h}\right|_{1, h}^{2} \\
\geq & 2 \alpha C_{3}\left\|v_{h}\right\|^{2}+\alpha\left(2 C_{2}-\alpha-2 C_{4} h^{2}-2 C h\right)\left|v_{h}\right|_{1, h}^{2},
\end{aligned}
$$

where we have used (3.27) in Hypothesis (H4) and the second inequality in (3.31). Choosing $h$ such that

$$
2 C_{2}-\alpha>2 C_{4} h^{2}+2 C h
$$

we have

$$
\left\|\boldsymbol{q}_{h}+A \nabla_{h} v_{h}\right\|^{2} \geq \inf _{\boldsymbol{s}_{h} \in X_{h}^{c}}\left\|\boldsymbol{s}_{h}+A \nabla_{h} v_{h}\right\|^{2} \geq C\left\|v_{h}\right\|_{1, h}^{2} .
$$

Using the triangle inequality, we get

$$
C\left\|\boldsymbol{q}_{h}\right\|^{2} \leq\left\|\boldsymbol{q}_{h}+A \nabla_{h} v_{h}\right\|^{2}+\left|v_{h}\right|_{1, h}^{2} \leq C\left\|\boldsymbol{q}_{h}+A \nabla_{h} v_{h}\right\|^{2},
$$

which completes the proof.

Corollary 3.1. Under Hypotheses (H1'), (H4) and (3.6), if $h$ is sufficiently small, then

$$
\begin{gathered}
L_{h}\left(v_{h}, \boldsymbol{q}_{h} ; v_{h}, \boldsymbol{q}_{h}\right) \geq C\left\{\left\|\boldsymbol{q}_{h}\right\|_{H(\mathrm{div} ; \Omega)}^{2}+\left\|v_{h}\right\|_{1, h}^{2}\right\}, \quad \forall\left(v_{h}, \boldsymbol{q}_{h}\right) \in U_{h} \times X_{h}^{c}, \\
\left\|u-u_{h}\right\|_{1, h}+\left\|\boldsymbol{p}-\boldsymbol{p}_{h}\right\|_{H(\mathrm{div} ; \Omega)} \leq C h\left\{\|u\|_{2}+\|\boldsymbol{p}\|_{2}\right\},
\end{gathered}
$$


where $(u, \boldsymbol{p}=-A \nabla u) \in\left(U \cap H^{2}(\Omega)\right) \times\left(X \cap\left(H^{2}(\Omega)\right)^{d}\right)$ and $\left(u_{h}, \boldsymbol{p}_{h}\right) \in U_{h} \times X_{h}^{c}$ are the exact and the finite element solutions, respectively.

Remark 3.3. Define

$$
X_{h}^{c}=\left\{\boldsymbol{q} \in X \cap\left(H^{1}(\Omega)\right)^{d} ; \boldsymbol{q}_{\mid K} \in\left(\mathcal{R}_{1}(K)\right)^{d}, \forall K \in \mathcal{T}_{h}\right\},
$$

where $\mathcal{R}_{1}(K)$ denotes $\mathcal{P}_{1}(K)$ (the space of linear polynomials) or $\mathcal{Q}_{1}(K)$ (the space of bilinear polynomials), while $U_{h}$ is still defined by (3.23). Then (H1') (cf. [11]), (H4) and (3.6) hold, where $I_{h}^{c}$ can be taken as the well-known Clément interpolation operator [21], 22].

Remark 3.4. Our method can be applied for other choices of $X_{h}$ and $U_{h}$. For example, on triangles, we define (cf. [1], [36], 19])

$$
\begin{aligned}
U_{h}=\left\{v \in L^{2}(\Omega) ; v_{\left.\right|_{K}}\right. & \in \mathcal{P}_{3}(K), K \in \mathcal{T}_{h} \\
& \left.\int_{e}[v] w=0, w \in \mathcal{P}_{2}(e), e \in S^{0}, \int_{e} v w=0, w \in \mathcal{P}_{2}(e), e \in S_{D}^{\partial}\right\},
\end{aligned}
$$

$$
\begin{gathered}
X_{h}=\left\{\boldsymbol{q} \in X ; \boldsymbol{q}_{\left.\right|_{K}} \in B D F M_{3}(K), R T N_{2}(K), K \in \mathcal{T}_{h}\right\}, \\
X_{h}^{c}=\left\{\boldsymbol{q} \in\left(H^{1}(\Omega)\right)^{2} \cap X ; \boldsymbol{q}_{\left.\right|_{K}} \in\left(\mathcal{P}_{2}(K)\right)^{2}, K \in \mathcal{T}_{h}\right\},
\end{gathered}
$$

it can be easily verified that Hypotheses (H1)-(H3) (or (H1'), (H4)) hold. Therefore, using similar arguments, we can obtain the coerciveness and the error bound $O\left(h^{3}\right)$ (or $O\left(h^{2}\right)$ ), with (3.44) and (3.45) (or (3.44) and (3.46)).

Remark 3.5. We can further consider the Robin-boundary value problem:

$$
\begin{array}{r}
-\operatorname{div}(A \nabla u)+\kappa u=f \quad \text { in } \Omega, \\
u=0 \quad \text { on } \Gamma_{D}, \\
\boldsymbol{n} \cdot A \nabla u+\rho u=0 \quad \text { on } \Gamma_{N},
\end{array}
$$

where if $\Gamma_{D}=\emptyset$, we require that either $\kappa(x)$ or $\rho(x)$ is bounded below away from zero; if $\Gamma_{D} \neq \emptyset$, we require that both $\kappa(x)$ and $\rho(x)$ are nonnegative functions. $A$ is a sufficiently smooth, symmetric matrix of coefficients which satisfies (2.2).

We introduce

$$
W_{0, N}(\Omega)=\left\{(\boldsymbol{p}, u) \in H(\operatorname{div} ; \Omega) \times U ;-\boldsymbol{p} \cdot \boldsymbol{n}+\rho u=0 \text { on } \Gamma_{N}\right\}
$$

where $W_{0, N}(\Omega)$ is a Hilbert space with respect to the norm $\|\boldsymbol{p}\|_{H(\mathrm{div} ; \Omega)}+\|u\|_{1}$ (cf. [23]).

We consider the following finite element method: Find $\left(u_{h}, \boldsymbol{p}_{h}\right) \in W_{h} \subset W_{0, N}(\Omega)$ such that

$$
\left(\boldsymbol{p}_{h}+A \nabla_{h} u_{h}, \boldsymbol{q}_{h}+A \nabla_{h} v_{h}\right)+\left(\operatorname{div} \boldsymbol{p}_{h}+\kappa u_{h}, \operatorname{div} \boldsymbol{q}_{h}+\kappa v_{h}\right)=\left(f, \operatorname{div} \boldsymbol{q}_{h}+\kappa v_{h}\right)
$$


for all $\left(v_{h}, \boldsymbol{q}_{h}\right) \in W_{h}$, where

$$
\begin{aligned}
& W_{h}=\left\{(\boldsymbol{q}, v) \in H(\operatorname{div} ; \Omega) \times L^{2}(\Omega) ;\right. \\
& \boldsymbol{q}_{\left.\right|_{K}} \in X(K)\left(\text { or } \boldsymbol{q} \in\left(H^{1}(\Omega)\right)^{d} \text { and }\left(\mathcal{R}_{1}(K)\right)^{d}\right), \forall K \in \mathcal{T}_{h}, \\
& v_{\left.\right|_{K}} \in U(K), \forall K \in \mathcal{T}_{h}, \int_{e}[v]=0, e \in S^{0}, \int_{e} v=0, e \in S_{D}^{\partial}, \\
&\left.\boldsymbol{q} \cdot \boldsymbol{n}=\rho v \text { on } S_{N}^{\partial}\right\} .
\end{aligned}
$$

Similarly, we can obtain the coerciveness and the optimal error bound $O(h)$.

\section{QUADRILATERAL ELEMENTS}

Clearly, under Hypotheses (H1)-(H3) (or (H1'), (H4) and (3.6)), we have established both coerciveness and error bound for the first-order system least-squares nonconforming mixed finite element problem (3.2). However, in the previous section we only dealt with triangular (or rectangular) elements in $\Re^{2}$, or tetrahedral (or rectangular solid) elements in $\Re^{3}$. In this section we will construct quadrilateral elements satisfying (H1)-(H3) (or (H1'), (H4) and (3.6)). Due to the Clément interpolation, (H4) can still be easily verified.

We consider the quadrilateral triangulation $\mathcal{T}_{h}=\{K\}$ in the $x y$-plane, with $K$ a quadrilateral whose diameter is $h_{K}$ and whose four vertices are $\left(x_{i}, y_{i}\right), 1 \leq i \leq 4$, and $h=\sup _{K \in \mathcal{T}_{h}} h_{K}$ is the mesh size. $|e|$ denotes the length of any edge $e \in \partial K$, and $\boldsymbol{n}_{e}$ denotes the unit outward normal vector to $e$. In addition, in this section the curl operator $\operatorname{curl} v=\left(-\frac{\partial v}{\partial y}, \frac{\partial v}{\partial x}\right)^{t}$ is used. $|K|$ denotes the measurement of $K$.

Let $\hat{K}$ be the reference square on the $\xi \eta$-plane, and let $F_{K}: \hat{K} \rightarrow K$ be the invertible mapping, with inverse mapping $F_{K}^{-1}: K \rightarrow \hat{K}$. From the assigned function $\hat{v}: \hat{K} \rightarrow \Re$ we can get a corresponding function $v_{K}: K \rightarrow \Re$ by $v_{K}(x, y):=$ $\hat{v}\left(F_{K}^{-1}(x, y)\right)$.

Lemma 4.1. For any given function $v \in H^{1}(K)$, if it has zero mean value over any given side $e \in \partial K\left(\int_{e} v=0\right)$, or over $K\left(\int_{K} v=0\right)$, then, under the shape regular condition of $\mathcal{T}_{h}$,

$$
\|v\|_{0, K} \leq C h_{K}|v|_{1, K} .
$$

Proof. Since the restriction of $F_{K}$ to any given side $e \in \partial K$ is an affine mapping, from $\int_{e} v=0$ we know that $\int_{\hat{e}} \hat{v}=0$. Then, applying the Poincaré inequality [14] to the reference element $\hat{K}$, we have $\|\hat{v}\|_{0, \hat{K}} \leq C|\hat{v}|_{1, \hat{K}}$. As a result, $\|v\|_{0, K} \leq C h_{K}\|\hat{v}\|_{0, \hat{K}} \leq C h_{K}|\hat{v}|_{1, \hat{K}} \leq C h_{K}|v|_{1, K}$. If $\int_{K} v=0$, it follows from the Poincaré inequality again that $\|v\|_{0, K} \leq C h_{K}|v|_{1, K}$.

4.1. A quadrilateral nonconforming element. Define

$$
\begin{gathered}
Y(K)=\operatorname{span}\left\{1, x, y, B_{K}(x, y)\right\}, \quad B_{K}(x, y)=\left(\xi^{2}-\eta^{2}\right) \circ F_{K}^{-1}, \\
U_{h}=\left\{v \in L^{2}(\Omega) ; v_{\left.\right|_{K}} \in Y(K), K \in \mathcal{T}_{h},\right. \\
\left.\int_{e}[v]=0, e \in S^{0}, \int_{e} v=0, e \in S_{D}^{\partial}\right\} .
\end{gathered}
$$


Remark 4.1. Unlike [18], here $Y(K)$ is not a parametric or nonparametric space, but a part-parametric one, and this allows for not only arbitrary shape-regular quadrilateral meshes, but also efficient calculation on the reference element $\hat{K}$.

Theorem 4.1. If $w \in H^{2}(K)$, we can define a unique $I_{K} w \in Y(K)$ such that

$$
\int_{e}\left(I_{K} w-w\right)=0 \quad \forall e \in \partial K,
$$

where $I_{K} w$ satisfies

$$
\begin{gathered}
\left\|w-I_{K} w\right\|_{0, K}+h_{K}\left|w-I_{K} w\right|_{1, K} \leq C h_{K}^{2}\|w\|_{2, K}, \\
h_{K}^{2 m-2} \| w-\left.I_{K} w\right|_{m, K}+\left|I_{K} w\right|_{1, K} \leq C|w|_{1, K} \quad(m=0,1) .
\end{gathered}
$$

Proof. To determine $I_{K} w \in Y(K)$, we only need to show that the following coefficient matrix is nonsingular:

$$
\left(\begin{array}{cccc}
\left|e_{1}\right|^{-1} \int_{e_{1}} & \left|e_{1}\right|^{-1} \int_{e_{1}} x & \left|e_{1}\right|^{-1} \int_{e_{1}} y & \left|e_{1}\right|^{-1} \int_{e_{1}} B_{K}(x, y) \\
\left|e_{2}\right|^{-1} \int_{e_{2}} & \left|e_{2}\right|^{-1} \int_{e_{2}} x & \left|e_{2}\right|^{-1} \int_{e_{2}} y & \left|e_{2}\right|^{-1} \int_{e_{2}} B_{K}(x, y) \\
\left|e_{3}\right|^{-1} \int_{e_{3}} & \left|e_{3}\right|^{-1} \int_{e_{3}} x & \left|e_{3}\right|^{-1} \int_{e_{3}} y & \left|e_{3}\right|^{-1} \int_{e_{3}} B_{K}(x, y) \\
\left|e_{4}\right|^{-1} \int_{e_{4}} & \left|e_{4}\right|^{-1} \int_{e_{4}} x & \left|e_{4}\right|^{-1} \int_{e_{4}} y & \left|e_{4}\right|^{-1} \int_{e_{4}} B_{K}(x, y)
\end{array}\right)
$$

where $1 \leq i(\bmod 4) \leq 4$

$$
\begin{gathered}
\left|e_{i}\right|^{-1} \int_{e_{i}}=1, \quad\left|e_{i}\right|^{-1} \int_{e_{i}} B_{K}=(-1)^{i} \frac{2}{3}, \\
\left|e_{i}\right|^{-1} \int_{e_{i}} x=\frac{x_{i}+x_{i+1}}{2}, \quad\left|e_{i}\right|^{-1} \int_{e_{i}} y=\frac{y_{i}+y_{i+1}}{2} .
\end{gathered}
$$

It can be seen that the determinant of (4.7) is $\frac{4|K|}{3} \neq 0$, which leads to the conclusion.

To show both (4.5) and (4.6), we let $I_{K} w(x, y)=a_{1}+a_{2} x+a_{3} y+a_{4} B_{K}(x, y)$ on each $K$, where the $a_{i}, 1 \leq i \leq 4$, are coefficients to be determined, and we let $b_{i}=\int_{e_{i}} w /\left|e_{i}\right|, e_{i} \in \partial K, 1 \leq i \leq 4$. By solving a standard algebraic linear system, we get

$$
\begin{aligned}
a_{4} & =\frac{3}{8}\left(b_{4}-b_{3}+b_{2}-b_{1}\right), \\
\left(\begin{array}{c}
a_{2} \\
a_{3}
\end{array}\right) & =\frac{1}{4|K|}\left(\begin{array}{cc}
y_{4}-y_{2} & y_{1}-y_{3} \\
x_{2}-x_{4} & x_{3}-x_{1}
\end{array}\right)\left(\begin{array}{c}
b_{2}-b_{1}+b_{3}-b_{4} \\
b_{3}-b_{2}+b_{4}-b_{1}
\end{array}\right),
\end{aligned}
$$

where $a_{1}=b_{1}-\frac{x_{1}+x_{2}}{2} a_{2}-\frac{y_{1}+y_{2}}{2} a_{3}+\frac{2}{3} a_{4}$.

Note that

$$
\int_{K} \nabla\left(w-I_{K} w\right)=\int_{\partial K}\left(w-I_{K} w\right)=0 .
$$

From Lemma 4.1 we have

$$
\begin{aligned}
& \left|w-I_{K} w\right|_{1, K} \leq C h_{K}\left|w-I_{K} w\right|_{2, K} \\
& \quad \quad\left(\text { with } \nabla\left(w-I_{K} w\right) \text { vanishing average on } K\right), \\
& \left\|w-I_{K} w\right\|_{0, K} \leq C h_{K}\left|w-I_{K} w\right|_{1, K}
\end{aligned}
$$

(with $w-I_{K} w$ vanishing average on $\partial K$ ). 
In what follows, we shall show that

$$
\left|I_{K} w\right|_{r, K} \leq C|w|_{r, K} \quad(r=1,2) .
$$

By an easy but tedious calculation, from the standard trace theorem [20, [22] we have

$$
\left|I_{K} w\right|_{1, K} \leq C\left\{\sum_{i=1}^{4}\left(\left|e_{i}\right|^{-1} \int_{e_{i}} w\right)^{2}\right\}^{1 / 2} \leq C\|\hat{w}\|_{1, \hat{K}} .
$$

Due to the fact that for any constant polynomial $p_{0} \in \mathcal{P}_{0}(K)$ the function $\tilde{v} \in Y(K)$ defined by $w+p_{0}$ is $I_{K} w+p_{0}$, we have

$$
\left|I_{K} w\right|_{1, K}=|\tilde{v}|_{1, K} \leq C \inf _{p_{0} \in \mathcal{P}_{0}(K)}\left\{\left\|\hat{w}+p_{0}\right\|_{0, \hat{K}}+|\hat{w}|_{1, \hat{K}}\right\} \leq C|\hat{w}|_{1, \hat{K}} \leq C|w|_{1, K} .
$$

Next, similarly to (4.13), from (4.8) and using the trace theorem again, we get

$$
\left|I_{K} w\right|_{2, K} \leq C h_{K}^{-1}\left\{\sum_{i=1}^{4}\left(\left|e_{i}\right|^{-1} \int_{e_{i}} w\right)^{2}\right\}^{1 / 2} \leq C h_{K}^{-2}\left\{\|w\|_{0, K}+h_{K}|w|_{1, K}\right\} .
$$

Also, since for any linear polynomial $p_{1} \in \mathcal{P}_{1}(K)$ the function $\tilde{v} \in Y(K)$ defined by $w+p_{1}$ is $I_{K} w+p_{1}$, we have

$$
\left|I_{K} w\right|_{2, K}=|\tilde{v}|_{2, K} \leq C h_{K}^{-2} \inf _{p_{1} \in \mathcal{P}_{1}(K)}\left\{\left\|w+p_{1}\right\|_{0, K}+h_{K}\left|w+p_{1}\right|_{1, K}\right\} .
$$

Furthermore, it can be seen that

$$
\begin{aligned}
\inf _{p_{1} \in \mathcal{P}_{1}(K)}\left\|w+p_{1}\right\|_{0, K} & \leq \inf _{p_{1} \in \mathcal{P}_{1}(K)} \inf _{p_{0} \in \mathcal{P}_{0}(K)}\left\|w+p_{1}+p_{0}\right\|_{0, K} \\
& \leq C h_{K} \inf _{p_{1} \in \mathcal{P}_{1}(K)}\left|w+p_{1}\right|_{1, K}, \\
\inf _{p_{1} \in \mathcal{P}_{1}(K)}\left|w+p_{1}\right|_{1, K} & =\inf _{p_{1} \in \mathcal{P}_{1}(K)}\left\|\nabla w+\nabla p_{1}\right\|_{0, K} \\
& \leq\left\|\nabla w+\boldsymbol{p}_{0}\right\|_{0, K} \leq C h_{K}|\nabla w|_{1, K},
\end{aligned}
$$

where we have chosen a special linear polynomial $p_{1}=d_{0}+d_{1} x+d_{2} y \in \mathcal{P}_{1}(K)$ with $\boldsymbol{p}_{0}=\left(d_{1}, d_{2}\right)^{t}=-\int_{K} \nabla w /|K|$; that is to say, $\nabla w+\boldsymbol{p}_{0}$ has a vanishing average on $K$, and the above estimation is derived from Lemma 4.1.

Therefore, we have

$$
\inf _{p_{1} \in \mathcal{P}_{1}(K)}\left\{\left\|w+p_{1}\right\|_{0, K}+h_{K}\left|w+p_{1}\right|_{1, K}\right\} \leq C h_{K}^{2}|w|_{2, K} .
$$

It follows that $\left|I_{K} w\right|_{2, K} \leq C|w|_{2, K}$. So (4.10)-(4.12) yield both (4.5) and (4.6).

Remark 4.2. It is interesting to note that both (4.5) and (4.6) are derived from the standard Poincaré inequality, not from the Bramble-Hilbert lemma (cf. [20], [22]). As a matter of fact, since $\mathcal{P}_{1}(K) \circ F_{K}$ is only a proper subspace of $\mathcal{Q}_{1}(\hat{K})$ and $Y(K)$ is not an isoparametric space, the classical estimation on quadrilaterals is not available. 
Corollary 4.1. Define $I_{h}: U \cap H^{2}(\Omega) \rightarrow U_{h}$ as follows:

$$
I_{h} w(x, y)=I_{K} w(x, y) \quad \forall(x, y) \in K, \quad \forall K \in \mathcal{T}_{h} .
$$

Then

$$
|| I_{h} w-w||+h\left|w-I_{h} w\right|_{1, h} \leq C h^{2}\|w\|_{2},
$$

$$
\left(\sum_{K \in \mathcal{T}_{h}} h_{K}^{2 m-2}\left\|w-I_{h} w\right\|_{m, K}^{2}\right)^{1 / 2}+\left|I_{h} w\right|_{1, h} \leq C|w|_{1} \quad(m=0,1) .
$$

4.2. A quadrilateral normal continuous element. Define

$D(K)=\operatorname{span}\left\{(1,0)^{t},(0,1)^{t},(x, y)^{t}, \operatorname{curl}\left(N_{1} \circ F_{K}^{-1}\right)\right\}, \quad N_{1}(\xi, \eta)=(1+\xi)(1+\eta) / 4$,

$$
X_{h}=\left\{\boldsymbol{q} \in X ; \boldsymbol{q}_{\left.\right|_{K}} \in D(K), K \in \mathcal{T}_{h}\right\} .
$$

Remark 4.3. $D(K)$ is a new element for quadrilaterals, but is the same as the $\mathrm{RT}_{[0]}$ rectangular element [5], 19] when $\mathcal{T}_{h}$ is composed of rectangles.

Theorem 4.2. Given a function $\chi \in H^{1}(\operatorname{div} ; K)$, we can find a unique interpolant $\Pi_{K} \chi \in D(K)$ such that

$$
\begin{gathered}
\int_{e}\left(\Pi_{K} \boldsymbol{\chi}-\boldsymbol{\chi}\right) \cdot \boldsymbol{n}_{e}=0 \quad \forall e \in \partial K, \\
\left\|\Pi_{K} \boldsymbol{\chi}-\boldsymbol{\chi}\right\|_{0, K} \leq C h_{K}|\boldsymbol{\chi}|_{1, K}, \\
\left\|\operatorname{div}\left(\boldsymbol{\chi}-\Pi_{K} \boldsymbol{\chi}\right)\right\|_{0, K} \leq C h_{K}|\operatorname{div} \boldsymbol{\chi}|_{1, K}, \\
\operatorname{div} \Pi_{K} \boldsymbol{\chi}=P_{K} \operatorname{div} \boldsymbol{\chi}, \quad\left\|\operatorname{div} \Pi_{K} \boldsymbol{\chi}\right\|_{0, K} \leq\|\operatorname{div} \boldsymbol{\chi}\|_{0, K},
\end{gathered}
$$

where $P_{K}$ is the $L^{2}$-projection operator onto $\mathcal{P}_{0}(K)$, the space of constant polynomials.

Proof. To show the existence and uniqueness of $\Pi_{K} \chi$, we only show that the matrix generated from (4.23) is nonsingular. Let the outward unit normal vector to $e_{i}$ be denoted by $\boldsymbol{n}_{e_{i}}=\left(y_{i}-y_{i+1}, x_{i+1}-x_{i}\right)^{t} /\left|e_{i}\right|$; then from (4.23) we get the following coefficient matrix:

$$
\left(\begin{array}{cccc}
y_{1}-y_{2} & x_{2}-x_{1} & y_{1} x_{2}-y_{2} x_{1} & 1 \\
y_{2}-y_{3} & x_{3}-x_{2} & y_{2} x_{3}-y_{3} x_{2} & 0 \\
y_{3}-y_{4} & x_{4}-x_{3} & y_{3} x_{4}-y_{4} x_{3} & 0 \\
y_{4}-y_{1} & x_{1}-x_{4} & y_{4} x_{1}-y_{1} x_{4} & -1
\end{array}\right)
$$

By a direct calculation, we know that the determinant of (4.27) is

$$
\left|\begin{array}{ll}
y_{1}-y_{3} & x_{3}-x_{1} \\
y_{2}-y_{4} & x_{4}-x_{2}
\end{array}\right|\left|\begin{array}{ll}
y_{2}-y_{3} & x_{3}-x_{2} \\
y_{2}-y_{4} & x_{4}-x_{2}
\end{array}\right| \neq 0,
$$

since under the shape regular condition we have

$$
\left|\begin{array}{llll}
x_{1} & y_{1} & 1 & 1 \\
x_{2} & y_{2} & 1 & 0 \\
x_{3} & y_{3} & 1 & 0 \\
x_{4} & y_{4} & 1 & 0
\end{array}\right| \neq 0
$$

Next, we consider (4.24). 
Consider two adjacent sides of $K$, say $e_{1}, e_{2}$, with outward unit vectors $\boldsymbol{n}_{e_{1}}, \boldsymbol{n}_{e_{2}}$. Under the shape regular condition, $\boldsymbol{n}_{e_{1}}, \boldsymbol{n}_{e_{2}}$ are linearly independent, and they can form a base in the plane. Then, any given vector-valued function $\chi$ can be uniquely written as $\boldsymbol{\chi}=\left(\boldsymbol{\chi} \cdot \boldsymbol{n}_{e_{1}}\right) \boldsymbol{n}_{e_{1}}+\left(\boldsymbol{\chi} \cdot \boldsymbol{n}_{e_{2}}\right) \boldsymbol{n}_{e_{2}}$, and it can be easily seen that

$$
\|\chi\|_{E} \leq\left|\chi \cdot \boldsymbol{n}_{e_{1}}\right|+\left|\chi \cdot \boldsymbol{n}_{e_{2}}\right| \leq 2\|\chi\|_{E}
$$

where $\|\cdot\|_{E}$ is the norm in 2-dimensional Euclidean space. Since $\Pi_{K} \chi$ is defined by (4.23), i.e.,, $\left(\Pi_{K} \boldsymbol{\chi}-\boldsymbol{\chi}\right) \cdot \boldsymbol{n}_{e_{i}}$ has a vanishing average on $e \in \partial K$, by Lemma 4.1 we have

$$
\|\left.\left(\Pi_{K} \chi-\chi\right) \cdot \boldsymbol{n}_{e_{i}}\right|_{0, K} \leq C h_{K}\left|\left(\Pi_{K} \chi-\chi\right) \cdot \boldsymbol{n}_{e_{i}}\right|_{1, K} \leq C h_{K}\left|\Pi_{K} \chi-\chi\right|_{1, K} .
$$

Then

$$
\begin{aligned}
\left\|\Pi_{K} \chi-\chi\right\|_{0, K} & \leq C\left\{\left\|\left(\Pi_{K} \chi-\chi\right) \cdot \boldsymbol{n}_{e_{1}}\right\|_{0, K}+\left\|\left(\Pi_{K} \chi-\chi\right) \cdot \boldsymbol{n}_{e_{2}}\right\|_{0, K}\right\} \\
& \leq C h_{K}\left\{\left|\left(\Pi_{K} \chi-\chi\right) \cdot \boldsymbol{n}_{e_{1}}\right|_{1, K}+\left|\left(\Pi_{K} \chi-\chi\right) \cdot \boldsymbol{n}_{e_{2}}\right|_{1, K}\right\} \\
& \leq C h_{K}\left|\Pi_{K} \chi-\chi\right|_{1, K} .
\end{aligned}
$$

Now we will show that

$$
\left|\Pi_{K} \chi\right|_{1, K} \leq C|\chi|_{1, K} .
$$

To that goal, define $b_{i}=\int_{e_{i}} \boldsymbol{\chi} \cdot \boldsymbol{n}_{e_{i}}, 1 \leq i \leq 4$, and $\Pi_{K} \boldsymbol{\chi}=(a, b)^{t}+c \boldsymbol{\varphi}_{K}+d \boldsymbol{\omega}_{K}$, with $\boldsymbol{\varphi}_{K}=(x, y)^{t}$ and $\boldsymbol{\omega}_{K}=\operatorname{curl}\left(N_{1} \circ F_{K}^{-1}\right)$. From (4.23) we know that for any constant vector $\boldsymbol{p}_{0} \in\left(\mathcal{P}_{0}(K)\right)^{2}$ we have $\Pi_{K} \boldsymbol{p}_{0} \equiv \boldsymbol{p}_{0}$, and so

$$
\begin{gathered}
|c| \leq C h_{K}^{-1}\left|\sum_{i=1}^{4} b_{i}\right|=C h_{K}^{-1}\left|\int_{K} \operatorname{div} \chi\right| \leq C\|\operatorname{div} \boldsymbol{\chi}\|_{0, K}, \\
|d| \leq C \sum_{i=1}^{4}\left|b_{i}\right| \leq C h_{K}|| \hat{\chi} \|_{1, \hat{K}} \leq C h_{K}\left\{\left\|\hat{\chi}+p_{0}\right\|_{0, \hat{K}}+|\hat{\chi}|_{1, \hat{K}}\right\} \\
\leq C h_{K}|\hat{\chi}|_{1, \hat{K}} \leq C h_{K}|\chi|_{1, K} .
\end{gathered}
$$

Noting that

$$
\left|\boldsymbol{\varphi}_{K}\right|_{1, K} \leq C h_{K}, \quad\left|\boldsymbol{\omega}_{K}\right|_{1, K} \leq C\left|N_{1} \circ F_{K}^{-1}\right|_{2, K} \leq C h_{K}^{-1},
$$

we have

$$
\left|\Pi_{K} \chi\right|_{1, K} \leq|c|\left|\boldsymbol{\varphi}_{K}\right|_{1, K}+|d|\left|\boldsymbol{\omega}_{K}\right|_{1, K} \leq C|\chi|_{1, K} .
$$

Obviously, (4.24) follows from (4.29) and (4.30).

Moreover, from (4.23) and $\operatorname{div} \Pi_{K} \chi \in \mathcal{P}_{0}(K)$, we have

$$
\int_{K}\left(\operatorname{div} \chi-P_{K} \operatorname{div} \chi\right)=\int_{K} \operatorname{div}\left(\chi-\Pi_{K} \chi\right)=0,
$$

where $P_{K}$ is the standard orthogonal $L^{2}$-projection operator onto $\mathcal{P}_{0}(K)$. Thus both (4.25) and (4.26) hold. The proof is finished.

Corollary 4.2. Let $\Pi_{h}: H^{1}(\operatorname{div} ; \Omega) \rightarrow X_{h}$ be defined by

$$
\Pi_{h} \chi(x, y)=\Pi_{K} \chi(x, y) \quad \forall(x, y) \in K, \quad \forall K \in \mathcal{T}_{h} .
$$


Then

$$
\begin{gathered}
\left\|\Pi_{h} \chi-\chi\right\| \leq C h|\chi|_{1}, \quad\left\|\operatorname{div}\left(\Pi_{h} \chi-\chi\right)\right\| \leq C h|\operatorname{div} \chi|_{1}, \\
\operatorname{div} \Pi_{h} \chi=P_{h} \operatorname{div} \chi, \quad\left\|\operatorname{div} \Pi_{h} \chi\right\| \leq\|\operatorname{div} \chi\|,
\end{gathered}
$$

where $P_{h}$ is the standard othogonal $L^{2}$-pojection operator onto $M_{h}$ defined by

$$
M_{h}=\left\{v \in L^{2}(\Omega) ; v_{\left.\right|_{K}} \in \mathcal{P}_{0}(K), \forall K \in \mathcal{T}_{h}\right\} .
$$

Lemma 4.2. For any $s \in D(K)$,

$$
\operatorname{div} \boldsymbol{s}_{\left.\right|_{K}}=\text { constant }, \quad \boldsymbol{s} \cdot \boldsymbol{n}_{e}=\text { constant }, \quad \forall e \in \partial K .
$$

Proof. Since the restrictions of $N_{1} \circ F_{K}^{-1}$ to $e_{2}$ and $e_{3}$ are zero, it follows that

$$
\operatorname{curl}\left(N_{1} \circ F_{K}^{-1}\right) \cdot \boldsymbol{n}_{e_{2}}=\operatorname{curl}\left(N_{1} \circ F_{K}^{-1}\right) \cdot \boldsymbol{n}_{e_{3}}=0 \quad \text { on } e_{2} \text { and } e_{3} \text {. }
$$

Next, let us consider, say, $e_{4}$, with the unit normal vector $\boldsymbol{n}_{e_{4}}$ and the unit tangent vector $\boldsymbol{\tau}_{e_{4}}$. Since

$$
\operatorname{curl}\left(N_{1} \circ F_{K}^{-1}\right) \cdot \boldsymbol{n}_{e_{4}}=\frac{\partial\left(N_{1} \circ F_{K}^{-1}\right)}{\partial \boldsymbol{\tau}_{e_{4}}}
$$

and the restriction to $e_{4}$ of $N_{1} \circ F_{K}^{-1}$ is a linear polynomial, we immediately know that $\operatorname{curl}\left(N_{1} \circ F_{K}^{-1}\right) \cdot \boldsymbol{n}_{e_{4}}=$ constant on $e_{4}$.

Remark 4.4. Lemma 4.2 indicates that the interpolation $\Pi_{K} \chi$ can be also determined by the mid-point values $\boldsymbol{\chi} \cdot \boldsymbol{n}$ on the four sides of $\partial K$.

Corollary 4.3. There exists a constant $C>0$, independent of $h$, such that

$$
\sup _{\chi \in X_{h}} \frac{(v, \operatorname{div} \boldsymbol{\chi})}{\|\chi\|_{H(\operatorname{div} ; \Omega)}} \geq C\|v\|, \quad \forall v \in M_{h} .
$$

Proof. For any given $v \in M_{h}$, let $s \in\left(H^{1}(\Omega)\right)^{2}$ be such that

$$
\operatorname{div} s=v, \quad\|s\|_{1}+\|s\|_{H(\operatorname{div} ; \Omega)} \leq C\|v\| .
$$

We can define $\Pi_{h} s \in X_{h}$ by

$$
\int_{e}\left(\boldsymbol{s}-\Pi_{h} \boldsymbol{s}\right) \cdot \boldsymbol{n}_{e}=0, \quad \forall e \in \partial K, \quad \forall K \in \mathcal{T}_{h},
$$

with

$$
\left\|\Pi_{h} \boldsymbol{s}\right\| \leq C|\boldsymbol{s}|_{1}, \quad\left\|\operatorname{div} \Pi_{h} \boldsymbol{s}\right\| \leq\|\operatorname{div} \boldsymbol{s}\| .
$$

Therefore, we get

$$
\begin{aligned}
\sup _{\boldsymbol{\chi} \in X_{h}} \frac{(v, \operatorname{div} \boldsymbol{\chi})}{\|\chi\|_{H(\operatorname{div} ; \Omega)}} & \geq \frac{\left(v, \operatorname{div} \Pi_{h} \boldsymbol{s}\right)}{\left\|\Pi_{h} \boldsymbol{s}\right\|_{H(\operatorname{div} ; \Omega)}} \\
& =\frac{(v, \operatorname{div} \boldsymbol{s})}{\left\|\Pi_{h} \boldsymbol{s}\right\|_{H(\operatorname{div} ; \Omega)}}+\frac{\left(v, \operatorname{div}\left(\Pi_{h} \boldsymbol{s}-\boldsymbol{s}\right)\right)}{\left\|\Pi_{h} \boldsymbol{s}\right\|_{H(\operatorname{div} ; \Omega)}} \\
& =\frac{(v, \operatorname{div} \boldsymbol{s})}{\left\|\Pi_{h} \boldsymbol{s}\right\|_{H(\operatorname{div} ; \Omega)}} \geq C\|v\| . \quad \square
\end{aligned}
$$

Remark 4.5. Corollaries 4.2 and 4.3 indicate that $\left(X_{h}, M_{h}\right)$ can be used in the classical mixed finite element approximation for (2.5) of second-order elliptic problems, when using arbitrary quadrilateral meshes. 
Remark 4.6. It is obvious that Hypotheses (H1)-(H3) hold with $U_{h}$ and $X_{h}$ defined by (4.3) and (4.22), respectively. Therefore, on the arbitrary quadrilateral triangulation, we can easily establish the coerciveness and the error bound $O(h)$ with both (4.3) and (4.22) used for solving (3.2).

Remark 4.7. For other quadrilateral versions of the $\mathrm{RTN}_{[0]}$ rectangular mixed element, (4.38) and the second inequality in (4.37) do not hold, since the Piolatransformation is involved (cf. [19], 25]). In the literature, (4.38) is referred to as the property of commuting diagram. This property is very important (cf. [19], 35], [37, 38]).

In what follows, we give other properties of $X_{h}$ on quadrilateral meshes. To do so, we first define

$$
V_{h}=\left\{v \in H^{1}(\Omega) ; v \circ F_{K} \in \mathcal{Q}_{1}(\hat{K}), K \in \mathcal{T}_{h}, v_{\left.\right|_{\Gamma_{N}}}=0\right\},
$$

where $\mathcal{Q}_{1}(\hat{K})=\operatorname{span}\left\{N_{i}(\xi, \eta), 1 \leq i \leq 4\right\}$ is the space of bilinear polynomials on $\hat{K}$, with $N_{i}(\xi, \eta)=\left(1+\xi_{i} \xi\right)\left(1+\eta_{i} \eta\right) / 4,\left(\xi_{i}, \eta_{i}\right) \in\{(1,1),(-1,1),(-1,-1),(1,-1)\}$.

Theorem 4.3. We have

$$
\operatorname{curl} V_{h} \subset X_{h} \text {. }
$$

Proof. For any given $v \in V_{h}$ we have $v_{\left.\right|_{K}}=\sum_{i=1}^{4} v_{i} N_{i} \circ F_{K}^{-1}$, with the $v_{i}, 1 \leq i \leq 4$, being nodal values of $v$. To show (4.47), we only need to show that

$$
\operatorname{curl}\left(N_{i} \circ F_{K}^{-1}\right) \in \operatorname{span}\left\{(1,0)^{t},(0,1)^{t}, \operatorname{curl}\left(N_{1} \circ F_{K}^{-1}\right)\right\} .
$$

Noting that

$$
1=\sum_{i=1}^{4} N_{i} \circ F_{K}^{-1}, \quad x=\sum_{i=1}^{4} x_{i} N_{i} \circ F_{K}^{-1}, \quad y=\sum_{i=1}^{4} y_{i} N_{i} \circ F_{K}^{-1},
$$

we have

$$
\left\{\begin{array}{l}
\sum_{i=1}^{4} \operatorname{curl}\left(N_{i} \circ F_{K}^{-1}\right)=(0,0)^{t} \\
\sum_{i=1}^{4} x_{i} \operatorname{curl}\left(N_{i} \circ F_{K}^{-1}\right)=(0,1)^{t}, \\
\sum_{i=1}^{4} y_{i} \operatorname{curl}\left(N_{i} \circ F_{K}^{-1}\right)=(-1,0)^{t},
\end{array}\right.
$$

which can alternatively be written as

$$
\left\{\begin{aligned}
\sum_{i=2}^{4} \operatorname{curl}\left(N_{i} \circ F_{K}^{-1}\right) & =-\operatorname{curl}\left(N_{1} \circ F_{K}^{-1}\right), \\
\sum_{i=2}^{4} x_{i} \operatorname{curl}\left(N_{i} \circ F_{K}^{-1}\right) & =(0,1)^{t}-x_{1} \operatorname{curl}\left(N_{1} \circ F_{K}^{-1}\right), \\
\sum_{i=2}^{4} y_{i} \operatorname{curl}\left(N_{i} \circ F_{K}^{-1}\right) & =(-1,0)^{t}-y_{1} \operatorname{curl}\left(N_{1} \circ F_{K}^{-1}\right) .
\end{aligned}\right.
$$

By virtue of the shape-regularity of the partition, we know that

$$
\left(\begin{array}{lll}
1 & 1 & 1 \\
x_{2} & x_{3} & x_{4} \\
y_{2} & y_{3} & y_{4}
\end{array}\right)
$$


is a nonsingular matrix, and by solving (4.50) we get

$$
\operatorname{curl}\left(N_{i} \circ F_{K}^{-1}\right)=\left(a_{i}, d_{i}\right)^{t}+g_{i} \operatorname{curl}\left(N_{1} \circ F_{K}^{-1}\right), \quad 2 \leq i \leq 4,
$$

with $\left(a_{i}, d_{i}, g_{i}\right)$ being constants. It immediately follows that (4.47) is true.

Corollary 4.4. For any $s \in X_{h} \operatorname{div} s=0$, there is an $v \in V_{h}$ such that

$$
s=\operatorname{curl} v \text {. }
$$

\section{Conclusions}

In this paper, in fact, we have shown that both coerciveness and optimal error bounds in energy norms still hold for the first-order system least-squares mixed method for second-order elliptic problems subject to various homogeneous boundary conditions, even if a nonconforming finite element is used for the displacement and a conforming element for the stress, since, in most cases, the nonconforming finite element space $U_{h}$ satisfies Hypothesis (H1), while Hypotheses (H3) and (H1') can be derived from (H1) (cf. [11], [27]), and Hypotheses (H2) and (H4) are trivial in the standard interpolation theory.

On arbitrary regular quadrilaterals [20], we have constructed two new elements. One is a new variant of the rotated $\mathcal{Q}_{1}$ nonconforming rectangular element, and the other is a new variant of the lowest-order $\mathrm{RTN}_{[0]}$ rectangular element. These two elements can also be used for other problems, such as the Stokes problem and the Reissner-Mindlin plate problem (cf. [38). is

This new nonconforming element can be easily generalized to 3-D. The analogy

$$
Y(K)=\operatorname{span}\left\{1, x, y, z, B_{1 K}, B_{2 K}\right\}
$$

with $B_{1 K}(x, y, z)=\left(\xi^{2}-\eta^{2}\right) \circ F_{K}^{-1}, B_{2 K}(x, y, z)=\left(\xi^{2}-\zeta^{2}\right) \circ F_{K}^{-1}$. However, it seems difficult to construct an analogous normal continuous element in 3-D.

\section{ACKNowledgments}

The authors wish to thank the anonymous referees for many constructive comments that improved the paper greatly.

\section{REFERENCES}

[1] Z. Cai, R. Lazarov, T.A. Manteuffel and S.F. McCormick, First order system least-squares for second order partial differential equations: Part 1, SIAM J.Numer.Anal., 31(1994), pp.17851799. MR 95i:65133

[2] Z. Cai, T.A. Manteuffel and S.F. McCormick, First order system least-squares for second order partial differential equations: Part 2, SIAM J.Numer.Anal., 34(1997), pp.425-454. MR 98m:65039

[3] A.I. Pehlivanov, G.F. Carey and R.D. Lazarov, Least-squares mixed finite element methods for second order elliptic problems, SIAM J.Numer.Anal., 31(1994),pp.1368-1374. MR 95f:65206

[4] P.B. Bochev and M.D. Gunzburger, Finite element methods of least-squares type, SIAM Rev., 40(91998), pp.789-834. MR 99k:65104

[5] P.A. Raviart and J.M. Thomas, A mixed finite element method for second order elliptic problems, in: Mathematical Aspects of The Finite Element Method, Lecture Notes in Mathematics 606, Springer-Verlag, Berlin and New York, 1977, pp. 292-315. MR 58:3547

[6] F. Brezzi, J. Douglas Jr., M. Fortin and L.D. Marini, Efficient rectangular mixed finite elements in tw and three space variables, M2AN Math.Model. Anal.Numer., 21(1987), pp.581604. MR 88j:65249 
[7] F. Brezzi, J. Douglas Jr. and L.D. Marini, Two families of mixed finite elements for second order elliptic problems, Numer.Math., 47(1985), pp.217-235. MR 87g:65133

[8] J.C. Nédélec, Mixed finite elements in $\Re^{3}$, Numer.Math.,22(1985), pp.315-341. MR 81k:65125

[9] A new family of mixed finite elements in $\Re^{3}$, Numer.Math., 50(1986), pp.57-81. MR 88e:65145

[10] T. Arbogast and Zhang-xin Chen, On the implementation of mixed methods as nonconforming methods for second order elliptic problems, Math.Comput., 64(1995), pp.943-974.

[11] M. Crouzeix and P.A. Raviart, Conforming and nonconforming finite element methods for solving the stationary Stokes equations, RAIRO Anal.Numer., 7(1973), pp.33-74. MR 95k:65102

[12] Han Hou-de, Nonconforming elements in the mixed finite element method, J. Comput. Math. 2(1984), pp.223-234. MR 87d:65130

[13] J. Douglas, J. J. Santos, D. Sheen and X. Ye, Nonconforming Galerkin methods based on quadrilateral elements for second order elliptic problems, RAIRO, Math. Model and Numer. Anal, 33(1999), 747-770. MR 2000k:65206

[14] L.E. Payne and H.F. Weinberger, An optimal Poincaré inequality for convex domains, Archive for Rational Mechanics and Analysis, 5(1960), pp.286-294. MR 22:8198

[15] P. Kloucek and F. R. Toffoletto, The three dimensional nonconforming finite element solution of the Chapman-Ferraro problem, J. Comp. Phy. 150(1999), pp. 549-560. MR 99m:86009

[16] P. Kloucek, Bo Li and M. Luskin, Analysis of a class of nonconforming finite elements for crystalline microstructures, Math. Comput., 65(1996), 1111-1135. MR 97a:73076

[17] Bo Li and M. Luskin, Nonconforming finite element approximation of crystalline microstructures, Math. Comput. 67(1998), 917-944. MR 98j:73092

[18] R. Rannacher and S. Turek, Simple nonconforming quadrilateral Stokes element, Numerical Methods for Partial Differential Equations, 8(1992),pp.97-111. MR 92i:65170

[19] F. Brezzi and M. Fortin, Mixed and Hybrid Finite Element Methods, Springer-Verlag.(1991) MR 92d:65187

[20] P.G. Ciarlet, The Finite Element Method for Elliptic Problems, North-Holland, Amsterdam. (1978) MR 58:25001

[21] P. Clément, Approximation by finite elements using local regularization, RAIRO Anal.Numer. 8(1975),pp.77-84. MR 53:4569

[22] S.C. Brenner and L.R. Scott, The Mathematical Theory of Finite Element Methods, Texts Appl.Math.15, Springer-Verlag, New York, 1994. MR 95f:65001

[23] B. Lee, First order system least-squares for elliptic problems with Robin boundary conditions, SIAM J.Numer.Anal., 37(1999),pp.70-104. MR 2001b:65126

[24] G. Starke, Multilevel boundary functionals for least-squares mixed finite element methods, SIAM J.Numer.Anal., 36(1999),pp.1065-1074. MR 2000d:65220

[25] J. Wang and T. Mathew, Mixed finite element methods over quadrilaterals, in: Proceedings of 3rd Internat.Conference on Advances in Numerical Methods and Applications, J.T.Dimov, B.Sendov, and P.Vassilevski, eds, World Scientific, River Edge, NJ, 1994,pp.203-214. MR 2001f:65001

[26] J.H. Bramble, R.D. Lazarov and J.E. Pasciak, Least-squares for second order elliptic problems, Comput.Methods Appl.Mech.Engrg., 152(1998),pp.195-210. MR 99a:65145

[27] D.N. Arnold and R.S. Falk, A uniformly accurate finite element method for the ReissnerMindlin plate, SIAM J. Numer. Anal., 26(1989), pp.1276-1290. MR 91c:65068

[28] V. Girault and P.A. Raviart, Finite Element Methods for Navier-Stokes Equations: Theory and Algorithms, Springer-Verlarg, Berlin, New York, 1986. MR 88b:65129

[29] G. Strang and G.J. Fix, An Analysis of the Finite Element Method, Prentice-Hall, Englewood Cliffs, NJ, 1973. MR 56:1747

[30] F. Stummel, The generalized patch test, SIAM J.Numer.Anal., 16(1979), pp.449-471. MR 80e:65106

[31] A.I. Pehlivanov, G.F. Carey and P.S. Vassilevski, Least-squares mixed finite element methods for nonselfadjoint elliptic problems: I. Error estimates, Numer. Math., 72(1996), pp.501-522. MR 97f:65068

[32] D.N. Arnold and F. Brezzi, Mixed and nonconforming finite element methods: Implemenetation, postprocessing and error estimates, M2AN Math.Modelling Anal.Numer., 19(1985), pp.7-32. MR 87g:65126 
[33] R. Stenberg, Postprocessing schemes for some mixed finite elements, M2AN Math.Modelling Anal.Numer., 25(1991), pp. 151-167. MR 92a:65303

[34] R.A. Adams, Soblev Spaces, Academic Press, New York, 1975. MR 56:9247

[35] J. Douglas Jr. and J.E. Roberts, Global estimates for mixed methods for second order elliptic equations, Math. Comput., 44(1985), pp. 39-52. MR 86b:65122

[36] M. Crouzeix and R.S. Falk, Nonconforming finite elements for the Stokes problem, Math. Comput., 52(1989), pp. 437-456. MR 89i:65113

[37] D. Boffi, Fortin operator and discrete compactness for edge elements, Numer.Math., 87(2000), pp. 229-246. MR 2001k:65168

[38] Huoyuan Duan, Studies On Mixed Finite Element Methods, Ph.D. thesis, Institute of Mathematics, Academy of Mathematics and System Sciences, Chinese Academy of Sciences, Beijing, P.R. China, March, 2002.

Institute of Mathematics, Chinese Academy of Sciences, Beijing 100080, Peoples RePUBLIC OF CHINA

E-mail address: dhymath@yahoo.com.cn

Institute of Mathematics, Chinese Academy of Sciences, Beijing 100080, Peoples RePUBLIC OF CHINA

E-mail address: lin@fegen.com 